\title{
A Stackelberg game for the Italian tax evasion problem
}

\author{
Gianfranco Gambarelli ${ }^{1}$. Daniele Gervasio ${ }^{2}$. Francesca Maggioni ${ }^{1}$ (i) . \\ Daniel Faccini ${ }^{3}$
}

Received: 21 June 2021 / Accepted: 6 September 2021 / Published online: 1 October 2021

(c) The Author(s) 2021

\begin{abstract}
In this paper, we consider the problem of tax evasion, which occurs whenever an individual or business ignores tax laws. Fighting tax evasion is the main task of the Economic and Financial Military Police, which annually performs fiscal controls to track down and prosecute evaders at national level. Due to limited financial resources, the tax inspector is unable to audit the population entirely. In this article, we propose a model to assist the Italian tax inspector (Guardia di Finanza, G.d.F.) in allocating its budget among different business clusters, via a controller-controlled Stackelberg game. The G.d.F. is seen as the leader, while potential evaders are segmented into classes according to their business sizes, as set by the Italian regulatory framework. Numerical results on the real Italian case for fiscal year 2015 are provided. Insights on the optimal number of controls the inspector will have to perform among different business clusters are discussed and compared to the strategy implemented by the G.d.F.
\end{abstract}

Keywords Game theory · Stackelberg games · Resource allocation · Inspection games

Francesca Maggioni

francesca.maggioni@unibg.it

Gianfranco Gambarelli

gianfranco.gambarelli@unibg.it

Daniele Gervasio

daniele.gervasio@unibg.it

Daniel Faccini

daniel.faccini@unibg.it

1 Department of Economics, University of Bergamo, Bergamo, Italy

2 Department of Management, University of Bergamo, Bergamo, Italy

3 Department of Management, Information \& Production Engineering, University of Bergamo, Bergamo, Italy 


\section{Introduction}

In Italy, the problem of tax evasion is a well known and uncontrolled phenomenon, with estimates on total evaded amount settling around $11 \%$ of the national Gross Domestic Product (GDP) (Ministero dell'Economia 2020). The government tries to tackle evasion via different strategies, emphasizing for instance the development of supportive taxpaying values among citizens, via informational strategies, or through coercive policies (Keith 1990). In this last regard, the role played by the Economic \& Financial Military Police (Guardia di Finanza, G.d.F. from now on) is of significant relevance, enabling every year to recover a large amount of undeclared taxes via fiscal audits. Given the budget assigned to the G.d.F. by the Ministry of Economy and Finance, establishing how many controls the G.d.F. should perform every year among different classes of taxpayers is a concern of national relevance.

In this work we propose a Stackelberg game (Von Stackelberg 2010) to assist the Italian tax inspector (G.d.F.) in allocating its budget among different business clusters. In a Stackelberg game, the leader commits to a strategy first, and then the follower optimizes its payoff considering the leader's chosen action. Given the Italian tax evasion framework, the G.d.F. (i.e., leader) will first commit to a budget allocation and, therefore, to perform a given number of fiscal controls per taxpayers class. After observing the G.d.F's decision, every taxpayers class (i.e., the follower) will then choose its own evasion strategy. Our goal is to find the optimal mixed strategy for the G.d.F., taking into account that every follower knows the leader's mixed strategy when choosing its own.

The paper is organized as follows. We start in Sect. 2 with a brief survey of related literature. Section 3 provides the problem description, while Sect. 4 presents the formal model underlying our analysis. Section 5 presents numerical experience on the Italian real case, and Sect. 6 concludes the article discussing the shortcomings and possible extensions of our research.

\section{Literature review}

In the literature, problems of tax evasion deterrence trace back to the pioneering work of Allingham and Sandmo (1972) and consequent extensions by, among others, Andreoni et al. (1998), Kolm (1973), Srinivasan (1973), and Yitzhaklt (1974), all applications of Becker's seminal contribution on the theory of crime (Becker 1968). The proposed rational models of deterrence highly rely on decision theory and are based on the assumption that a potential criminal faces a fixed probability of being discovered (and consequently punished) when committing a crime. The potential criminal will therefore transgress if the anticipated benefits are greater than the predicted penalties and two factors influence this decision. The first is the likelihood with which the crime will be discovered, the second is the severity of the punishment. An increase in either of these two dimensions is then assumed to reduce the likelihood of the crime to be committed.

The claim that law compliance depends solely on the coercive power of the law defender (by means of controls and harsh punishments), nonetheless, has been ques- 
tioned by a number of authors. Above all, Graetz et al. (1986), Holler (1993), Tsebelis (1989), Tsebelis (1990a), Tsebelis (1990b), Wittman (1985) and Wittman (1993) highlighted that both the probability of committing a crime and the probability of discovering it should be treated as endogenous variables in crime deterrence models, hence paving the way to game theory in providing predictions on the interaction between crime and control rates. The game theoretic approach to crime deterrence is based on a two-player simultaneous-move game known as the inspection game. See (Avenhaus et al. 2002) for an extended summary on the variety of applications of inspection games. The first player (the would-be criminal) decides whether or not to violate a law, while the second player (the law defender) decides whether to inspect the other player or not. Inspection is assumed to be expensive, so the inspector prefers not to inspect if believing no crime will be committed. Compliance with the law is also expensive, which implies that if no inspection is carried out, the law is violated. The inspection game has a unique Nash equilibrium (Nash 1950) in mixed strategy which shows, as opposed to decision theoretic models and results, that an increase in the severity of penalties leaves the frequency of law infractions unchanged, with the only effect of reducing the frequency of inspections. See (Weissing and Ostrom 1991) for a deeper analysis on the general conditions under which changes in the payoffs of one player do not affect that player's behavior at equilibrium.

The simultaneous approach of the inspection game, as well as the need of the players to know each other to be rational enough to calculate their equilibrium probabilities, are somehow questionable in practical situations. Therefore, a first strand of literature (see Andreozzi 2004; Bianco et al. 1990; Cox 1994, and Hirshleifer and Rasmusen (1992)) relaxes the assumption of simultaneity and proposes a sequential version of the inspection game, in which the potential criminal chooses after observing the inspector's move. This is the so-called Stackelberg version of the game, plenty of successful real-life applications such as the placement of checkpoints and canine units at Los Angeles International airport (Pita et al. 2009), the choice of patrol routes for the US Coast Guard in Boston (Shieh et al. 2012), and the scheduling of fare inspections on Los Angeles Metro Rail system (Yin et al. 2012). Other famous examples are Jain et al. (2010), Jain et al. (2010), and Pita et al. (2009) applied to security. A second strand of literature relaxes the assumption of rationality and is based on evolutionary game theory (Weibull 1997). In Andreozzi (2002), Andreozzi (2010), Cressman et al. (1998), Katsikas et al. (2016) authors present models where agents are not assumed to be rational, rather they adapt their behavior over time from less profitable strategies to more profitable ones.

Given the previous researches on the subject, it is evident that the tax evasion problem applied to the Italian framework has not been addressed yet via a Stackelberg game. On this purpose, in the following, the Italian tax dodger's dilemma will be modeled and solved via a non-simultaneous dynamic game. 


\section{Problem description and notation}

We consider the tax inspector (the G.d.F.) which partitions the taxpayers into classes ${ }^{1}$ $i \in \mathcal{I}$, each having a private conflict with the unique controller and each treated as a single follower (see Borm et al. 1996). Formally, let:

$$
\mathcal{N}_{t}:=\left\{1, \ldots, n_{t}\right\}
$$

be the population of taxpayers at year time $t$ and let $\left\{\mathcal{N}_{t}^{i} \mid i \in \mathcal{I}\right\}$ with $\left|\mathcal{N}_{t}^{i}\right|=n_{t}^{i}$ be a disjoint partition of $\mathcal{N}_{t}$ into $I$ classes of taxpayers, such that $\sum_{i \in \mathcal{I}} n_{t}^{i}=n_{t}$. The goal of this section is to provide a model able to settle the optimal number of controls that the tax inspector (G.d.F.) will have to perform on every single class $i \in \mathcal{I}$ in order to maximize its total payoff, in compliance with the Italian regulations.

At the beginning of every year $t$, the Ministry of Economy and Finance assigns to the controller (G.d.F.) a budget $\mathcal{B}_{t}$ for the strategic objective of fighting tax evasion. However, only the portion $\mathcal{C}_{t}$ devoted to physical fiscal controls has to be considered as direct cost. The leader (G.d.F.) establishes how to divide the assigned budget $\mathcal{C}_{t}$ to support the costs of the inspections among the classes, meeting the minimum number of controls $v_{t}^{i}$ set by law for each of the groups. Besides undeclared taxes $\tau_{t}^{i}$ of class $i \in \mathcal{I}$ in year $t$, administrative penalties $\rho_{t}^{i}$ for the verified tax evasion of follower $i \in \mathcal{I}$ and the tax compliance ${ }^{2} \kappa_{t}^{i}$ should be considered. The information on the division chosen by the leader is made public and, consequently, each class chooses its evasion rate $e_{t}^{i}$. The controller goal is to determine -in the first step- the optimal number of fiscal controls $x_{t}^{i}$ to be performed for every class $i \in \mathcal{I}$ so as to maximize its total payoff. The following notation is assumed

\begin{tabular}{ll} 
Sets & \\
$\mathcal{I}:=\{1, \ldots, I\}$ & Set of classes; \\
$\mathcal{N}_{t}:=\left\{1, \ldots, n_{t}\right\}$ & Population of taxpayers at year $t ;$ \\
$\mathcal{N}_{t}^{i}:=\left\{1, \ldots, n_{t}^{i}\right\}$ & Population of taxpayers at year $t$ of class $i \in \mathcal{I}$. \\
Parameters & Overall budget assigned in year $t$ to fight tax evasion; \\
$\mathcal{B}_{t}$ & Portion of budget $\mathcal{B}_{t}$ directly assigned in year $t$ to fiscal controls \\
$\mathcal{C}_{t}$ & $\left(\mathcal{C}_{t} \leq \mathcal{B}_{t}\right) ;$ \\
& Portion of $\mathcal{C}_{t}$ assigned to fiscal controls of class $i \in \mathcal{I}$ in year $t ;$ \\
$\mathcal{C}_{t}^{i}$ & Minimum number of fiscal controls to be planned for class $i \in \mathcal{I}$ \\
$v_{t}^{i}$ & in year $t ;$ \\
$c_{t}^{i}$ & Unitary cost of planned fiscal controls for class $i \in \mathcal{I}$ in year $t ;$ \\
$\tau_{t}^{i}$ & Undeclared taxes of follower $i \in \mathcal{I}$ in year $t ;$ \\
$\hat{t}$ & Number of years to verify tax evasion; \\
\hline
\end{tabular}

\footnotetext{
1 We assume that followers do not share information and they play independently.

2 Tax compliance is the fulfillment to tax liability paid to reduce the tax gap.
} 


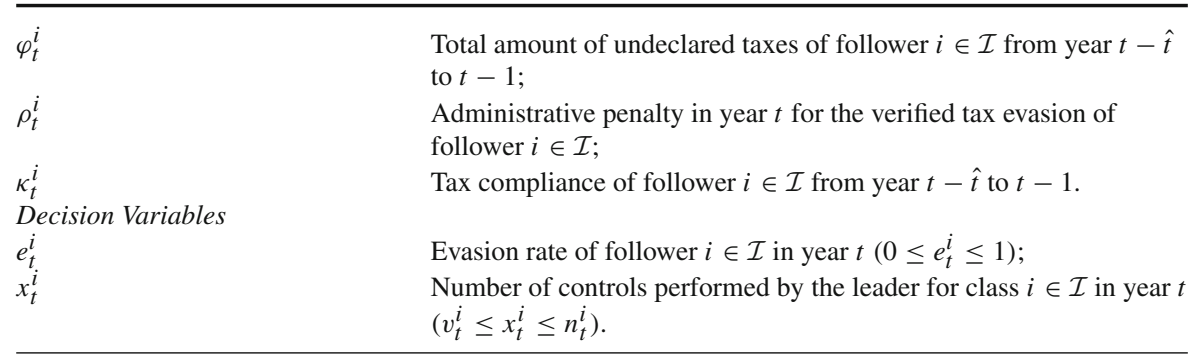

The following assumptions are made.

- The unitary cost $c_{t}^{i}$ of planned fiscal controls for class $i \in \mathcal{I}$ in year $t$ will be equal to the average unitary cost of the controls performed in year $t-1$ for class $i \in \mathcal{I}$ :

$$
c_{t}^{i}:=\frac{\mathcal{C}_{t-1}^{i}}{\bar{x}_{t-1}^{i}}
$$

where $\bar{x}_{t-1}^{i}$ denotes the number of actual controls performed by the leader in year $t-1$.

- The total amount of undeclared taxes of follower $i \in \mathcal{I}$ in year $t$ is computed as follows:

$$
\tau_{t}^{i}:=\operatorname{unIVA}_{t}^{i}+\operatorname{unIRAP}_{t}^{i}+\operatorname{unIRES}_{t}^{i}+\operatorname{unIRPEF}_{t}^{i},
$$

with unIVA $_{t}^{i}$, $\operatorname{unIRAP}_{t}^{i}$, $\operatorname{unIRES}_{t}^{i}$, unIRPEF $_{t}^{i}$ being respectively the undeclared value added tax ( $\operatorname{unIVA}_{t}^{i}$ ), regional income tax (unIRAP $\left.t_{t}^{i}\right)$, corporate income tax ( $\operatorname{unIRES}_{t}^{i}$ ), and personal income tax $\left(\operatorname{unIRPEF}_{t}^{i}\right)$ of follower $i \in \mathcal{I}$ in year $t$. Data on undeclared taxes are made public every year by the Ministry of Economy and Finance in the "Report on the Unobserved Economy and Social Security Evasion", see (Ministero dell'Economia 2020).

- For a given class $i \in \mathcal{I}$ and a year $t$, the total amount of undeclared taxes from year $t-\hat{t}$ to $t-1$ is computed as follows:

$$
\varphi_{t}^{i}:=\sum_{j=t-\hat{t}}^{t-1} \tau_{j}^{i} .
$$

- Whenever a tax evasion perpetrated by the follower $i \in \mathcal{I}$ is verified, administrative penalties are set in the amount of:

$$
\rho_{t}^{i}:=1.90 \cdot \varphi_{t}^{i}
$$

Penalties (4) are derived by averaging rates of the current regulation, according to which administrative penalties must be set for amounts not less than $90 \%$ and up to $180 \%$ of the undeclared taxes $\varphi_{t}^{i}$. For the sake of completeness, we acknowledge that actual regulations also enforce criminal penalties in the form of detentions for tax evasion. Specifically: 
- for fraudulent misrepresentation (i.e., false documents) the custodial sentence is applied if the undeclared tax $\tau_{t}^{i}$ exceeds 30,000 euros, or if the undeclared tax base $\beta_{t}^{i}$ exceeds $5 \%$ of revenues and income, and in any case $\beta_{t}^{i}$ exceeds 1,500,000 euros of revenues and income;

- for unfaithful declarations (i.e., without false documents) the custodial sentence is applied if the undeclared tax $\tau_{t}^{i}$ exceeds 150,000 euros, or if the undeclared tax base $\beta_{t}^{i}$ exceeds $10 \%$ of revenues and proceeds, and in any case $\beta_{t}^{i}$ exceeds 3,000,000 euros of revenues and proceeds;

- for omitted declarations the custodial sentence is applied if the undeclared tax $\tau_{t}^{i}$ exceeds 50,000 euros.

Furthermore, seizures for tax evasion are also prosecuted by the current regulation, in addition to administrative penalties. This measure consists in confiscating a property belonging to the convicted person and transferring it to the State. It can also consist, when the transfer of this property is not possible, in imposing on the convict the additional payment of a sum of money equivalent to the value of the property which should have been confiscated (the so called confiscation by equivalent). We choose not to reflect seizures and confiscations by equivalent value in our model, rather restricting to administrative penalties only.

\section{A Stackelberg game model}

In this section, we model the problem described in Sect. 3 as a Stackelberg game. We interpret the problem as an aggregation of private conflicts between two parties: the G.d.F. and each class of taxpayers. Therefore, the problem can be seen as a union of $I$ games in normal form, with the G.d.F. being the leader and groups of companies the followers. In Sect. 4.1, the players utility functions are derived, while in Sect. 4.2 the follower optimal evasion is obtained. Finally, in Sect. 4.3 the optimal number of fiscal controls are determined.

\subsection{Utility functions}

The leader (G.d.F.) must determine whether or not to perform a fiscal control in year $t$ to class $i \in \mathcal{I}$, while the taxpayer's possible pure strategies are to evade or not to evade. The probability that the leader will perform a control is denoted by $\frac{x_{t}^{i}}{n_{t}^{i}}$, while $e_{t}^{i}$ indicates the probability of the follower's evasion. If the leader performs a control in year $t$ on a follower $i \in \mathcal{I}$ who has decided to evade, then the follower will have to pay the undeclared tax $\tau_{t}^{i}$ as well as a penalty $\rho_{t}^{i}$ computed as in (4). Furthermore, independently from the evasion probability $e_{t}^{i}$, the evading follower will also have to pay the tax compliance $\kappa_{t}^{i}$. If, on the other hand, the leader does not perform a control in year $t$ on an evading follower $i \in \mathcal{I}$, then that player will gain the undeclared taxes $\tau_{t}^{i}$ as well as $\varphi_{t}^{i}$. Finally, players will have zero payoffs in all the remaining cases. See Table 1 . 
Table 1 Two-player normal form game in which the leader is the G.d.F. and the follower is one of the companies classes $i \in \mathcal{I}$

\begin{tabular}{lll}
\hline & Controlling $\left(\frac{x_{t}^{i}}{n_{t}^{i}}\right)$ & Not Controlling $\left(1-\frac{x_{t}^{i}}{n_{t}^{i}}\right)$ \\
\hline Evading $\left(e_{t}^{i}\right)$ & $\left(-\tau_{t}^{i}-\rho_{t}^{i}-\frac{\kappa_{t}^{i}}{e_{t}^{i}}, \tau_{t}^{i}+\rho_{t}^{i}+\frac{\kappa_{t}^{i}}{e_{t}^{i}}\right)$ & $\left(\tau_{t}^{i}+\varphi_{t}^{i},-\tau_{t}^{i}-\varphi_{t}^{i}\right)$ \\
Not Evading $\left(1-e_{t}^{i}\right)$ & $(0,0)$ & $(0,0)$ \\
\hline
\end{tabular}

It follows that for every follower $i \in \mathcal{I}$ the expected utility function in year $t$ is given by:

$$
f^{i}\left(e_{t}^{i}, x_{t}^{i}\right):=\left[e_{t}^{i}\left(\frac{x_{t}^{i}}{n_{t}^{i}}\right)\left(-\tau_{t}^{i}-\rho_{t}^{i}-\frac{\kappa_{t}^{i}}{e_{t}^{i}}\right)+e_{t}^{i}\left(1-\frac{x_{t}^{i}}{n_{t}^{i}}\right)\left(\tau_{t}^{i}+\varphi_{t}^{i}\right)\right],
$$

while the leader's expected utility functions are:

$$
l^{i}\left(e_{t}^{i}, x_{t}^{i}\right):=\left[e_{t}^{i}\left(\frac{x_{t}^{i}}{n_{t}^{i}}\right)\left(\tau_{t}^{i}+\rho_{t}^{i}+\frac{\kappa_{t}^{i}}{e_{t}^{i}}\right)+e_{t}^{i}\left(1-\frac{x_{t}^{i}}{n_{t}^{i}}\right)\left(-\tau_{t}^{i}-\varphi_{t}^{i}\right)\right] .
$$

The model is solved by backward induction. The leader considers what the best responses of the followers are, i.e., how class $i \in \mathcal{I}$ will respond after observing the quantity $x_{t}^{i}$ that the G.d.F. has set. The leader then picks the quantity that maximizes its payoff anticipating the predicted responses of the followers.

\subsection{Follower's optimal evasion}

The optimal evasion $e_{t}^{i *}$ of follower $i \in \mathcal{I}$ in year $t$, taking into account any arbitrary quantity $x_{t}^{i}$ chosen by the leader, is obtained solving the following one-dimension linear optimization problem:

$$
\begin{aligned}
\max _{0 \leq e_{t}^{i} \leq 1} f^{i}\left(e_{t}^{i}, x_{t}^{i}\right) & =\max _{0 \leq e_{t}^{i} \leq 1} e_{t}^{i}\left[\left(\frac{x_{t}^{i}}{n_{t}^{i}}\right)\left(-\tau_{t}^{i}-\rho_{t}^{i}-\frac{\kappa_{t}^{i}}{e_{t}^{i}}\right)+\left(1-\frac{x_{t}^{i}}{n_{t}^{i}}\right)\left(\tau_{t}^{i}+\varphi_{t}^{i}\right)\right] \\
& =\max _{0 \leq e_{t}^{i} \leq 1} e_{t}^{i}\left[\left(\frac{x_{t}^{i}}{n_{t}^{i}}\right)\left(-2 \tau_{t}^{i}-2.9 \varphi_{t}^{i}\right)+\tau_{t}^{i}+\varphi_{t}^{i}\right]-\frac{x_{t}^{i}}{n_{t}^{i}} \cdot \kappa_{t}^{i}
\end{aligned}
$$

Solving model (7), the optimal evasion $e_{t}^{i *}$ of follower $i \in \mathcal{I}$ is then given by:

$$
e_{t}^{i^{*}}:= \begin{cases}1 & \text { if } \quad x_{t}^{i}<n_{t}^{i} \cdot \frac{\tau_{t}^{i}+\varphi_{t}^{i}}{2 \tau_{t}^{i}+2.9 \varphi_{t}^{i}} \\ {[0,1] \quad \text { if } \quad x_{t}^{i}=n_{t}^{i} \cdot \frac{\tau_{t}^{i}+\varphi_{t}^{i}}{2 \tau_{t}^{i}+2.9 \varphi_{t}^{i}}} \\ 0 \quad \text { if } \quad x_{t}^{i}>n_{t}^{i} \cdot \frac{\tau_{t}^{i}+\varphi_{t}^{i}}{2 \tau_{t}^{i}+2.9 \varphi_{t}^{i}} .\end{cases}
$$




\subsection{Leader's optimal number of fiscal controls}

Knowing followers optimal evasions $e_{t}^{i *}$ given in (8) for $i \in \mathcal{I}$ at time $t$, the leader's utility functions (6) become the following piecewise linear functions:

$$
l^{i}\left(e_{t}^{i^{*}}, x_{t}^{i}\right):= \begin{cases}\frac{x_{t}^{i}}{n_{t}^{i}}\left(2 \tau_{t}^{i}+2.9 \varphi_{t}^{i}+\kappa_{t}^{i}\right)-\tau_{t}^{i}-\varphi_{t}^{i} & \text { if } x_{t}^{i}<n_{t}^{i} \cdot \frac{\tau_{t}^{i}+\varphi_{t}^{i}}{2 \tau_{t}^{i}+2.9 \varphi_{t}^{i}} \\ \frac{\tau_{t}^{i}+\varphi_{t}^{i}}{2 \tau_{t}^{i}+2.9 \varphi_{t}^{i}} \cdot \kappa_{t}^{i} & \text { if } x_{t}^{i}=n_{t}^{i} \cdot \frac{\tau_{t}^{i}+\varphi_{t}^{i}}{2 \tau_{t}^{i}+2.9 \varphi_{t}^{i}} \\ \frac{x_{t}^{i}}{n_{t}^{i}} \cdot \kappa_{t}^{i} & \text { if } \quad x_{t}^{i}>n_{t}^{i} \cdot \frac{\tau_{t}^{i}+\varphi_{t}^{i}}{2 \tau_{t}^{i}+2.9 \varphi_{t}^{i}}\end{cases}
$$

Therefore, the optimal number of fiscal controls that the leader will have to perform in year $t$ for every class $i \in \mathcal{I}$ to maximize its total payoff is obtained by solving the following integer optimization problem:

$$
\begin{aligned}
& \max _{x_{t}^{i}} \sum_{i \in \mathcal{I}} l^{i}\left(e_{t}^{i^{*}}, x_{t}^{i}\right) \\
& \text { s.t. } \quad \sum_{i \in \mathcal{I}} c_{t}^{i} \cdot x_{t}^{i} \leq \mathcal{C}_{t} \\
& v_{t}^{i} \leq x_{t}^{i} \leq n_{t}^{i} \quad i \in \mathcal{I} \\
& x_{t}^{i} \in \mathbb{N} \quad i \in \mathcal{I} .
\end{aligned}
$$

Objective function (9) expresses the maximization of the leader's total utility function. Constraint (10) represents the budget constraint, according to which the overall cost of fiscal controls performed by the leader must not exceed the available budget. Constraints (11) require the number of controls per class to be greater or equal to the minimum threshold set by law, and smaller than the number of taxpayers belonging to that class. Finally, constraints (12) define the decision variables to be integer.

\section{Numerical results}

The model formulated in Sect. 4 has been solved on a real case provided by Bergamo Economic \& Financial Military Police (Guardia di Finanza di Bergamo).

The computations have been performed on a 64-bit machine with 8 GB of RAM, a $1.8 \mathrm{GHz}$ Intel i7 processor, and numerical results are obtained under AMPL environment using CPLEX solver.

The year under investigation is $t=2015$. In quantifying undeclared taxes, we do not go back further than five years $(\hat{t}=5)$ because of the assessment limit set by the Italian legislation (article 43 DPR 600/73). This means that the tax inspector can check and fine undeclared taxes from year $t-5$ to year $t-1$. For year 2015, the overall budget assigned for the strategic objective of fighting tax evasion was $\mathcal{B}_{2015}=1,701,162,134$ euros, of which $\mathcal{C}_{2015}=629,430,000$ (approximately $37 \%$ of 
Table 2 Data concerning, for class $i=1,2,3$ in year 2015, the number of enterprises $n_{2015}^{i}$, the minimum number of fiscal controls to be planned $v_{2015}^{i}$, the unitary cost of planned fiscal controls $c_{2015}^{i}$, the undeclared taxes $\tau_{2015}^{i}$, the total amount of undeclared taxes $\varphi_{2015}^{i}$ from year $t-5$ to $t-1$, and the total amount of tax compliance $\kappa_{2015}^{i}$ from year $t-5$ to $t-1$

\begin{tabular}{lllllll}
\hline & $n_{2015}^{i}$ & $v_{2015}^{i}$ & $c_{2015}^{i}(€)$ & $\tau_{2015}^{i}(€)$ & $\varphi_{2015}^{i}(€)$ & $\kappa_{2015}^{i}(€)$ \\
\hline$i=1$ & $5,068,180$ & 7250 & 2840 & $28,846,000$ & $188,067,000$ & $26,873,000$ \\
$i=2$ & 51,376 & 5750 & 32,647 & $11,661,000$ & $74,718,000$ & $10,669,000$ \\
$i=3$ & 6252 & 234 & 266,560 & $24,889,000$ & $178,762,000$ & $25,558,000$ \\
\hline
\end{tabular}

$\mathcal{B}_{2015}$ ) are meant to perform fiscal controls. Following the assumptions of the previous section, we consider three classes of followers (i.e., $I=3$ ) partitioned according to the business size classification set by the Italian regulation in accordance with the European Union recommendation 2003/361 (see Ministero delle Attività Produttive 2005):

1. $\mathcal{N}_{t}^{1}$ is the set of taxpayers with annual turnover smaller than 5,165,000 euros (which are called small enterprises);

2. $\mathcal{N}_{t}^{2}$ is the set of taxpayers with annual turnover between 5,165,000 and 50,000,000 euros (which are called medium-sized enterprises);

3. $\mathcal{N}_{t}^{3}$ is the set of taxpayers with annual turnover greater than 50,000,000 euros (which are called large enterprises).

Values of the problem parameters are provided in Table 2. Specifically, for class $i=$ $1,2,3$ in year 2015, the first column refers to the number of enterprises $n_{2015}^{i}$, second column to the minimum number of fiscal controls to be planned $v_{2015}^{i}$, third column to the unitary cost of planned fiscal controls $c_{2015}^{i}$, forth column to the undeclared taxes $\tau_{2015}^{i}$, fifth column to the total amount of undeclared taxes $\varphi_{2015}^{i}$ from year $t-5$ to $t-1$, and finally last column the total amount of tax compliance $\kappa_{2015}^{i}$ from year $t-5$ to $t-1$. The values of $\varphi_{2015}^{i}$ and $\kappa_{2015}^{i}$ from $t-5=2010$ to $t-1=2014$ for every class $i \in \mathcal{I}$ are provided in Tables 3, 4. Notice that the unitary cost $c_{2015}^{i}$ of planned fiscal controls for class $i \in \mathcal{I}$ is computed by means of (1). However, since data about budget $\mathcal{C}_{t}^{i}$ and number of controls $\bar{x}_{t}^{i}$ performed in year $t=2014$ are not available, we use ex-post data about year $t=2015$. Specifically, we partition $\mathcal{C}_{2015}$ as follows:

$\mathcal{C}_{2014}^{1}=\mathcal{C}_{2014}^{2}=221,151,077 \approx 35 \% \cdot \mathcal{C}_{2015}, \quad \mathcal{C}_{2014}^{3}=187,127,834 \approx 30 \% \cdot \mathcal{C}_{2015}$

and set $\bar{x}_{2014}^{i}$ equal to the number of controls actually performed by the G.d.F. in year 2015, for all $i \in \mathcal{I}$, which are:

$$
\bar{x}_{2014}^{1}=\bar{x}_{2015}^{1}=77,897, \quad \bar{x}_{2014}^{2}=\bar{x}_{2015}^{2}=6,774 \quad \bar{x}_{2014}^{3}=\bar{x}_{2015}^{3}=702 .
$$

Solutions to problem (9)-(11) are:

$$
x_{2015}^{1^{*}}=7,259 \quad x_{2015}^{2^{*}}=5,756 \quad x_{2015}^{3^{*}}=1,579,
$$


Table 3 Undeclared taxes $\tau_{t}^{i}$ from year $t-5=2010$ to $t-1=2014$ per class $i \in \mathcal{I}$

\begin{tabular}{llllll}
\hline$\tau_{t}^{i}(€)$ & 2010 & 2011 & 2012 & 2013 & 2014 \\
\hline$i=1$ & $33,567,000$ & $38,419,000$ & $40,509,000$ & $37,552,000$ & $38,020,000$ \\
$i=2$ & $14,333,000$ & $16,406,000$ & $13,297,000$ & $15,620,000$ & $15,062,000$ \\
$i=3$ & $32,382,000$ & $37,063,000$ & $35,365,000$ & $37,085,000$ & $36,867,000$ \\
\hline
\end{tabular}

Table 4 Tax compliance $\kappa_{t}^{i}$ from year $t-5=2010$ to $t-1=2014$ per class $i \in \mathcal{I}$

\begin{tabular}{llllll}
\hline$\kappa_{t}^{i}(€)$ & 2010 & 2011 & 2012 & 2013 & 2014 \\
\hline$i=1$ & $4,432,000$ & $5,310,000$ & $5,679,000$ & $5,450,000$ & $6,002,000$ \\
$i=2$ & $1,893,000$ & $2,267,000$ & $1,864,000$ & $2,267,000$ & $2,378,000$ \\
$i=3$ & $4,275,000$ & $5,123,000$ & $4,957,000$ & $5,383,000$ & $5,820,000$ \\
\hline
\end{tabular}

which suggest that the leader should allocate 20,615,560 euros of its budget on small businesses, 187,916,132 euros on medium-sized businesses, and 420,898,240 euros on large businesses. The used budget amounts to $629,429,932$ euros, while the not used is only 68 euros, not enough to perform any further control. The number of controls to be performed on small businesses suggested by the solution seems to be the largest, however only $0.14 \%$ of the total number of taxpayers belonging to this class should be checked. On the other hand, the total number of controls that should be performed among taxpayers belonging to the medium-sized businesses is $11.20 \%$, while sets around $25.26 \%$ for large enterprises, showing the importance of controls on class $i=3$.

We finally compare the actual controls performed by the G.d.F. in year $t=2015$ given in (13) with the solutions reported in (14). The total number of controls actually performed by the G.d.F. in year 2015 among small businesses sets around $1.54 \%$ of the entire population $(i=1)$, around $13.19 \%$ for $i=2$, and around $11.23 \%$ for $i=3$. The model advocates to sensibly strengthen controls of class $i=3$ while relaxing controls of classes $i=1,2$. The radar plot shown in Fig. 1 reports a comparison of the overall number of controls divided into the three classes, respectively for the solution of model (9)-(11) (solid line) and the strategy actually performed by the G.d.F. in 2015 (dashed line).

\section{Conclusions}

In this paper, we considered the problem of tax evasion, faced by the Economic and Financial Military Police that annually performs fiscal controls. For this problem we proposed a model to assist the tax inspector in allocating its budget among different business classes, via a controller-controlled Stackelberg game. Numerical results on the real Italian case are provided for fiscal year 2015 to assist the Italian tax inspector (Guardia di Finanza, G.d.F.). The analysis showed that the G.d.F. should allocate a greater portion of its budget to control the class of large enterprises $\left(66.87 \%\right.$ of $\left.\mathcal{C}_{2015}\right)$, 


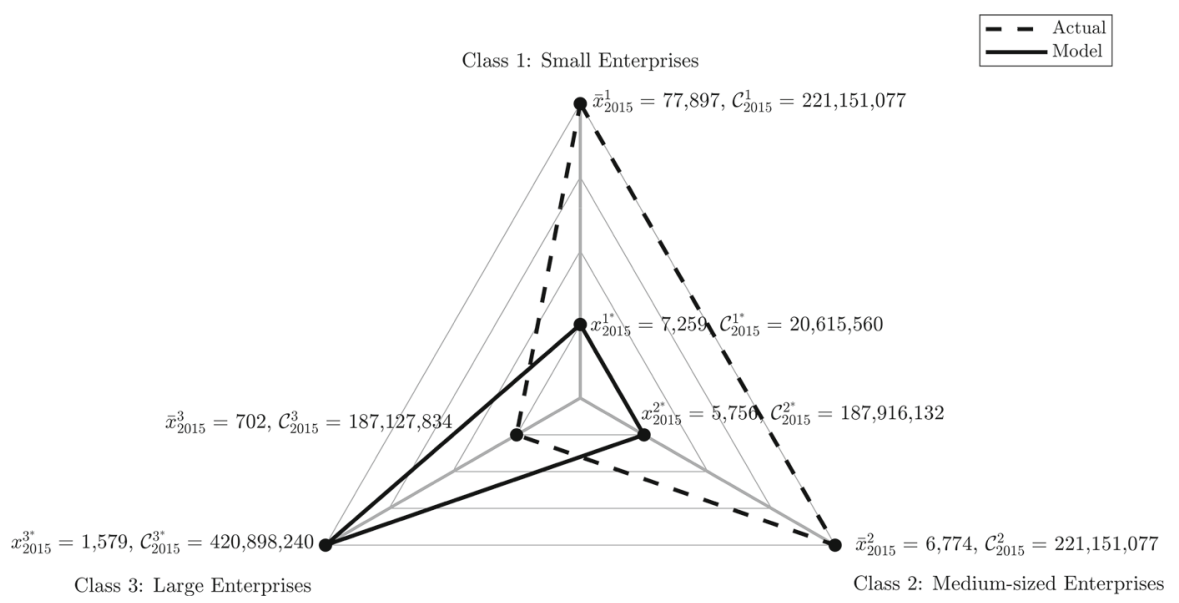

Fig. 1 Overall number of controls and total budget $\mathcal{C}_{2015}$ divided into classes $i \in \mathcal{I}$ obtained solving model (9)-(11) (solid line) and actually performed by the G.d.F. in year 2015 (dashed line)

while devoting $29.85 \%$ of $\mathcal{C}_{2015}$ to the medium-sized companies and only $3.27 \%$ of $\mathcal{C}_{2015}$ to small businesses. A comparison with the actual strategy implemented by the G.d.F. is finally performed, showing that the G.d.F. is currently investing more resources to control small businesses than the ones suggested by the model. Future works will consider an extension of the approach to include other tax years, taking into account novelty in the Italian legislation.

Acknowledgements We would like to thank Nicola Gatti and Martina Mori for their contribution on a preliminary version of this work. Claudia Rossi and Sabino Gervasio are also acknowledged for a description of the problem and the data provided.

Funding Open access funding provided by Università degli studi di Bergamo within the CRUI-CARE Agreement.

Open Access This article is licensed under a Creative Commons Attribution 4.0 International License, which permits use, sharing, adaptation, distribution and reproduction in any medium or format, as long as you give appropriate credit to the original author(s) and the source, provide a link to the Creative Commons licence, and indicate if changes were made. The images or other third party material in this article are included in the article's Creative Commons licence, unless indicated otherwise in a credit line to the material. If material is not included in the article's Creative Commons licence and your intended use is not permitted by statutory regulation or exceeds the permitted use, you will need to obtain permission directly from the copyright holder. To view a copy of this licence, visit http://creativecommons.org/licenses/by/4.0/.

\section{References}

Allingham MG, Sandmo A (1972) Income tax evasion: a theoretical analysis. J Public Econom 1(3-4):323338

Andreoni J, Erard B, Feinstein J (1998) Tax compliance. J Econom Literature 36(2):818-860

Andreozzi L (2002) Oscillations in against-pollution policies. an evolutionary analysis. Homo Oeconomicus 
Andreozzi L (2004) Rewarding policemen increases crime. another surprising result from the inspection game. Public choice 121(1-2): 69-82

Andreozzi L et al (2010) Inspection games with long-run inspectors. Euro J Appl Math 21(4-5):441-458

Avenhaus R, Von Stengel B, Zamir S (2002) Inspection games. Handbook Game Theory Econom Appl 3:1947-1987

Becker GS (1968) Crime and punishment: An economic approach. In: The economic dimensions of crime, pp. 13-68. Springer

Bianco WT, Ordeshook PC, Tsebelis G (1990) Crime and punishment: are one-shot, two-person games enough? Am Political Sci Rev 84(2):569-586

Borm P, Garcia-Jurado I, Potters J, Tijs S (1996) An amalgation of games. Euro J Operational Res 89(3):570580

Cox GW (1994) A note on crime and punishment. Public Choice 78(1):115-124

Cressman R, Morrison WG, Wen JF (1998) On the evolutionary dynamics of crime. Can J Econom, pp. 1101-1117

Graetz MJ, Reinganum JF, Wilde LL (1986) The tax compliance game: toward an interactive theory of law enforcement. J Law Econom Organ 2(1):1-32

Guardia di Finanza (G.d.F.). www.gdf.gov.it

Hirshleifer J, Rasmusen E (1992) Are equilibrium strategies unaffected by incentives? J Theor Politics 4(3):353-367

Holler MJ (1993) Fighting pollution when decisions are strategic. Public Choice 76(4):347-356

Jain M, Tsai J, Pita J, Kiekintveld C, Rathi S, Tambe M, Ordónez F (2010) Software assistants for randomized patrol planning for the lax airport police and the federal air marshal service. Interfaces 40(4):267-290

Jain M, Kardes E, Kiekintveld C, Ordóñez F, Tambe M (2010) Optimal defender allocation for massive security games: A branch and price approach. In: Workshop 25: Optimisation in Multi-agent Systems, p. 1

Katsikas S, Kolokoltsov V, Yang W (2016) Evolutionary inspection and corruption games. Games 7(4):31

Keith S (1990) Governmental policies to reduce tax evasion: coerced behavior versus services and values development. Policy Sci 23(1):57-72

Kolm SC (1973) A note on optimum tax evasion. J Public Econom 2(3):265-270

Ministero delle Attività Produttive: Adeguamento alla disciplina comunitaria dei criteri di individuazione di piccole e medie imprese. (2005)

Ministero dell'Economia e delle Finanze (MEF). www.mef.gov.it

Ministero dell'Economia e delle Finanze: Relazione sull'economia non osservata e sull'evasione fiscale e contributiva (2020)

Nash JF et al (1950) Equilibrium points in n-person games. Proc National Acad Sci 36(1):48-49

Pita J, Bellamane H, Jain M, Kiekintveld C, Tsai J, Ordónez F, Tambe M (2009) Security applications: lessons of real-world deployment. ACM SIGecom Exchanges 8(2):1-4

Pita J, Jain M, Ordónez F, Portway C, Tambe M, Western C, Paruchuri P, Kraus S (2009) Using game theory for los angeles airport security. AI Magazine 30(1):43-43

Shieh E, An B, Yang R, Tambe M, Baldwin C, DiRenzo J, Maule B, Meyer G (2012) Protect: A deployed game theoretic system to protect the ports of the united states. In: Proceedings of the 11th International Conference on Autonomous Agents and Multiagent Systems-Volume 1, pp. 13-20. Citeseer

Srinivasan TN (1973) Tax evasion: A model

Tsebelis G (1989) The abuse of probability in political analysis: The robinson crusoe fallacy. The American Political Science Review pp. 77-91

Tsebelis G (1990) Are sanctions effective? A game-theoretic analysis. J Conflict Resolut 34(1):3-28

Tsebelis G (1990) Penalty has no impact on crime: a game-theoretic analysis. Ration Soc 2(3):255-286

Von Stackelberg H (2010) Market structure and equilibrium. Springer, Berlin

Weibull JW (1997) Evolutionary game theory. MIT press, Cambridge

Weissing F, Ostrom E (1991) Crime and punishment: further reflections on the counterintuitive results of mixed equilibria games. J Theor Politics 3(3):343-350

Wittman D (1993) Nash equilibrium vs. maximin: a comparative game statics analysis. Euro J Political Econom 9(4):559-565

Wittman D (1985) Counter-intuitive results in game theory. Euro J Political Econom 1(1):77-89

Yin Z, Jiang AX, Johnson MP, Kiekintveld C, Leyton-Brown K, Sandholm T, Tambe M, Sullivan JP (2012) Trusts: Scheduling randomized patrols for fare inspection in transit systems. In: IAAI 
Yitzhaklt S (1974) A note on income tax evasion: a theoretical analysis. J Public Econom 3:201-202

Publisher's Note Springer Nature remains neutral with regard to jurisdictional claims in published maps and institutional affiliations. 